Received: 2015.06.10 Accepted: 2015.06.11 Published: 2015.XX.XX

\title{
Mycophenolic Acid Metabolites Acyl-Glucuronide and Glucoside Affect the Occurrence of Infectious Complications and Bone Marrow Dysfunction in Liver Transplant Recipients
}

\section{Authors' Contribution: Study Design A Data Collection B Statistical Analysis C Data Interpretation D Manuscript Preparation E Literature Search F Funds Collection G}

ABCDEF 1 Jolanta Zegarska*

ABCDEF 1 Ewa Hryniewiecka*

BCDEF 1 Dorota Żochowska

ABCDEF 2 Włodzimierz Tszyrsznic

BCDEF 2 Radosław Jaźwiec

BCDEF 2 Agnieszka Borowiec

BCDEF 2 Emilia Pawłowska

ABCDEFG 2 Michat Dadlez

ABCDEFG 1 Leszek Pączek
1 Department of Immunology, Transplant Medicine and Internal Diseases, Medical University of Warsaw, Transplantation Institute, Warsaw, Poland

2 Institute of Biochemistry and Biophysics Polish Academy of Sciences, Warsaw, Poland
Corresponding Author: Source of support:

* These 2 persons equally contributed to the presented work Leszek Pączek, e-mail: leszek.paczek@wum.edu.pl

This work was supported by the Polish National Center of Research and Development, grant No: NR13014410

Background: Mycophenolic acid (MPA) prodrugs are anti-proliferative immunosuppressive agents commonly used after organ transplantation. Although they are generally well tolerated by patients, adverse effects may occur. It is postulated that MPA metabolites could also contribute to these adverse effects.

Material/Methods: The objective of this study was the assessment of concentrations of total MPA and its metabolites, phenyl glucuronide (MPAG), acyl glucuronide (AcMPAG) and glucoside (GluMPA), using liquid chromatography combined with mass spectrometry (LC/MS/MS) in two groups: kidney transplant recipients and liver transplant patients. Associations of MPA and its metabolites with adverse effects were analyzed.

Results: $\quad$ The study group consisted of 211 recipients of liver or kidney transplants who received immunosuppressive therapy, including MPA prodrugs. Multivariant analysis showed a positive influence of MPA on gastroenterotoxicity in kidney transplant recipients. In liver patients, gastroenterotoxicity was associated with lower MPAG concentrations. A positive influence of AcMPAG on bacterial infections in liver transplant patients was observed. In liver transplant recipients, a positive influence of MPA and a negative influence of GluMPA levels on the PLT count were revealed. MPA and its metabolites did not influence the hemoglobin levels in both groups. There were no significant relationships among MPA, its metabolites and WBC counts.

Conclusions: In kidney transplant recipients, total MPA trough concentration is associated with gastroenterotoxicity and its monitoring could have important role in management of gastrointestinal complications. The quantification of AcMPAG in liver recipients receiving MPA may be helpful in avoiding bacterial infections. GluMPA seems to have a toxic effect on thrombopoiesis.

MeSH Keywords: Kidney Transplantation • Liver Transplantation • Mycophenolic Acid

Full-text PDF: http://www.annalsoftransplantation.com/abstract/index/idArt/894954

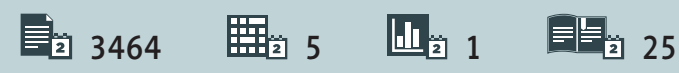




\section{Background}

Mycophenolic acid (MPA) is widely used for the treatment of patients undergoing solid organ transplantation as part of a

5 multiple drug regimen, usually with concomitant cyclosporine or tacrolimus and corticosteroids to prevent graft rejection. MPA blocks the conversion of inosine monophosphate by inosine monophosphate dehydrogenase (IMPDH), a key enzyme in the de novo purine biosynthetic pathway [1]. Although my-

10 cophenolic acid pro-drugs, including mycophenolate mofetil (MMF) and mycophenolate sodium (MPS), are generally welltolerated in patients, such adverse effects as: infections, leucopoenia, anemia, and gastrointestinal problems may occur, necessitating dose reduction or discontinuation and thereby

15 potentially jeopardizing patient and graft outcomes. Dose reduction and discontinuation of MPA therapy have been associated with an increased risk of acute rejection episodes and kidney graft loss [2].

20 In contrast to other immunosuppressants, mycophenolate mofetil and mycophenolate sodium preparations were introduced for clinical use without a recommendation of therapeutic drug monitoring (TDM). Clinical experience has shown that it is possible and, in some cases, necessary to use vari25 ous methods of TDM, such as the assessment of the area under the concentration-time curve (AUC) and minimum plasma drug concentration (through concentration). These evaluations have shown high inter- and intra-individual variability in the MPA exposure parameters. Although several MPA metabolites 30 have been identified, their assessment is not used routinely. The major MPA metabolite is phenolic glucuronide (MPAG), and other minor metabolites include 7-O-glucoside (GluMPA) and acyl-glucuronide (AcMPAG) [3,4]. Whereas MPAG is inactive, ACMPAG is capable of inhibiting human IMPDH in vitro 35 and has been considered toxic [4,5]. There is scarce information regarding GluMPA levels and actions in solid organ transplant (SOT) patients.

The aim of the study was to assess the levels of the total MPA 40 and of its three metabolites, MPAG, AcMPAG, and GluMPA, using liquid chromatography combined with tandem mass spectrometry (LC/MS/MS) in SOT recipients. We hypothesized that metabolites of MPA could affect the occurrence of the adverse effects of the drug.

45

\section{Material and Methods}

This study involved the participation of 211 solid organ trans50 plant recipients. All of the patients under the care of our transplant center were eligible for inclusion. All consecutive outpatients who gave their written informed consent to participate in 53 the study were included. All blood samples were taken during routine blood tests on outpatient visits to the Transplant and 1 Nephrology Clinic between November 2011 and July 2012. Blood was taken just before the administration of the morning dose of MPA, between 8.30 and 9.00 am (trough concentration) and after fasting for at least 8 hours. Blood was col- 5 lected in EDTA tubes and placed at $+4^{\circ} \mathrm{C}$, then centrifuged to obtain plasma. Plasma was stored at $-80^{\circ} \mathrm{C}$ until the time of determination of MPA and its metabolites concentrations by LC/MS/MS method. Blood sampling was accompanied by the collection of relevant laboratory and clinical data.

Renal function was assessed with the use of estimated glomerular filtration rate (eGFR) by Modification of Diet in Renal Disease (MDRD) [6]. MPA dosage: To standardize the data on daily mycophenolate mofetil and mycophenolate sodium dos- 15 age $\left(\mathrm{MPA}_{\mathrm{cd}}\right)$, mycophenolate sodium doses were converted to equivalent mycophenolate mofetil doses $\left(\mathrm{MPA}_{\mathrm{cd}}=\mathrm{MPS}\right.$ [mg/day] $\times 1.3889$ ). Gastroenterotoxicity in patients was assessed based on patients' medical history. Symptoms, including diarrhea, abdominal pain, and vomiting, especially recurrent vomiting, were 20 identified. We excluded other causes of these problems, such as CMV infection or other infections on the basis of fever incidence; elevated C-reactive protein levels; or positive CMV DNA PCR. According to the clinical symptoms, the patients were divided into two groups: those with gastroenterotoxicity (recur- 25 rent or few episodes of diarrhea, abdominal pain or vomiting, not related to infection, with the resolution of the symptoms following MPA dose reduction) and those without gastroenterotoxicity (no typical symptoms or symptoms related to infection). The incidence of infections was based on the pres- 30 ence of suggestive clinical characteristics and was confirmed by microbiologic studies of representative biological samples and agreeable results from additional tests, including elevated CRP, procalcitonin, and white blood cell counts. CMV infection was diagnosed on the basis of typical clinical symptoms and 35 positive CMV DNA PCR results. Anemia was diagnosed if the blood hemoglobin was $<120 \mathrm{~g} / \mathrm{L}$ (women) or $<130 \mathrm{~g} / \mathrm{L}$ (men).

Chemicals. The chemicals used included the following: LC-MS grade - methanol, $25 \%$ ammonium hydroxide and formic acid 40 (J.T. Baker), and analytical grade - ammonium acetate (POCh, Gliwice, Poland). Ultra-pure water was obtained from a water purification system (Mili-Q, Millipore, Milford, MA, USA). MPA, deuterated MPA (MPA-d3), AcMPAG, MPAG, and GluMPA (Toronto Research Chemicals Inc., North York, Canada) were a 45 gift from Roche Poland. Stock solutions were prepared in methanol and stored at $-20^{\circ} \mathrm{C}$. As an internal standard for MPA and all metabolites, MPA-d3 was applied.

Sample preparation. MPA and all metabolites were quanti- 50 fied in the blood plasma. The whole blood samples were centrifuged (10 min at 1000 RCF) to obtain the plasma. Sample preparation was performed as follows: $100 \mu \mathrm{L}$ of plasma was 53 
1 Table 1. Monitored transitions, cone voltages, collision energies, and retention times of the analyzed compounds.

\begin{tabular}{|c|c|c|c|c|c|}
\hline & Immunosuppressive agent & MRM transition & Cone voltage & Collision energy & $\begin{array}{l}\text { Retention } \\
\text { time [min] }\end{array}$ \\
\hline \multirow[t]{4}{*}{5} & \multirow{2}{*}{ Mycophenolic acid (MPA) } & $338.16>207.10$ (qt) & 10 & 15 & 1.81 \\
\hline & & $338.16>275.19$ & 10 & 15 & \\
\hline & \multirow{3}{*}{ MPA glucoside } & $500.21>303.02(q t)$ & 15 & 20 & 1.50 \\
\hline & & $500.21>275.10$ & 15 & 25 & \\
\hline \multirow[t]{4}{*}{10} & & $500.21>207.12$ & 15 & 30 & \\
\hline & \multirow{3}{*}{ MPA acyl glucuronide } & $514.19>207.12(q t)$ & 15 & 35 & 1.64 \\
\hline & & $514.19>303.02$ & 15 & 20 & \\
\hline & & $514.19>321.10$ & 15 & 10 & \\
\hline \multirow[t]{4}{*}{15} & \multirow{3}{*}{ MPA phenyl glucuronide } & $514.19>321.10(\mathrm{qt})$ & 15 & 10 & 1.33 \\
\hline & & $514.19>303.02$ & 15 & 20 & \\
\hline & & $514.19>207.12$ & 15 & 35 & \\
\hline & MPA-d3 & $341.22>210.04$ & 10 & 25 & 1.80 \\
\hline
\end{tabular}

20 qt - quantification transition; MPA - mycophenolic acid; MPA-d3 - deuterated mycophenolic acid; MRM - multiple-reaction monitoring 20 (MRM) mode.

transferred into a $1.5-\mathrm{mL}$ silanized conical test tube (Sigma Aldrich), and then $250 \mu \mathrm{L}$ of methanol (with MPA-d3) was add25 ed for protein precipitation and analyte extraction. After the mixture was vortexed (1 min) and centrifuged ( 2 min at 18626 $\mathrm{RCF})$, the entire supernatant was transferred to the vial and analyzed by LC/MS/MS.

30 Instrumentation. The instrumentation consisted of a Waters Acquity Ultra Performance Liquid Chromatograph coupled with a Waters TQ-S triple-quadruple mass spectrometer. For the instrument control and data acquisition, MassLynx software was used. LC/MS/MS analysis was performed in the positive elec-

35 trospray ionization mode (ESI). The mass spectrometer was operated in a multiple-reaction monitoring (MRM) mode. The concentration of each analyte was calculated per MPA-d3.

\section{Analyses}

40

For chromatographic separation, we applied the UPLC BEH Phenyl column $(50 \times 2.1 \mathrm{~mm}, 1.7 \mu \mathrm{m}$, Waters), thermostated at $45^{\circ} \mathrm{C}$. Mobile phase A consisted of $300 \mu \mathrm{L}$ of formic acid and $900 \mu \mathrm{L}$ of $25 \% \mathrm{NH}_{4} \mathrm{OH}$ in $1000 \mathrm{~mL}$ of water, and mobile phase $45 \mathrm{~B}$ consisted exclusively of methanol. The flow rate of the mobile phase was set at $0.5 \mathrm{~mL} / \mathrm{min}$, and the injection volume was $10 \mu \mathrm{L}$ for the analysis of MPA, GluMPA and AcMPAG and $2 \mu \mathrm{L}$ for the analysis of MPAG. The gradient scheme was 3\% B initially, followed by an increase to $90 \%$ B at $2.0 \mathrm{~min}$. At $2.3 \mathrm{~min}$,

50 the mobile phase reverted to the initial conditions ( $3 \% \mathrm{~B})$. The total analysis time was $3 \mathrm{~min}$, including re-equilibration time. For all of the analyzed compounds, the mass spectrometer 53 optimized settings were as follows: capillary voltage $=2.5 \mathrm{kV}$, desolvation temperature $=200^{\circ} \mathrm{C}$, desolvation gas flow $=800 \mathrm{~L} / \mathrm{h}$, cone gas flow $=150 \mathrm{~L} / \mathrm{h}$, nebulizer gas pressure $=7.0 \mathrm{bar}$, source temperature $=150^{\circ} \mathrm{C}$. The MRM transitions, cone voltages, colli- 25 sion energies and retention times used in the described methods are presented in Table 1. The first MRM transition of each compound served as a quantitative transition; the second, as a confirmation transition. To define the relationship between the concentration and detector responses of analytes, 6-level 30 calibrators were prepared for each MPA metabolite, as well as for the parent compound. The concentrations of the calibrators covered entire ranges of the expected (determined empirically based on several patients' samples prior to the validation process) concentrations in the patients' samples $(1-7 \mu \mathrm{g} / \mathrm{ml}$ for 35 MPA, $0.01-1 \mu \mathrm{g} / \mathrm{ml}$ for MPA glucoside, $0.5-5 \mu \mathrm{g} / \mathrm{ml}$ for AcMPAG and $10-200 \mu \mathrm{g} / \mathrm{ml}$ for MPAG). The mean R2 coefficients of the calibration curves for all compounds from 7 sample batches were not lower than 0.97 . The imprecision level of the method was assessed using 120 in-house control samples and was de- 40 termined by measuring 4 sets of 10 samples at three concentration levels within the ranges of expected concentration in the patient. Imprecision values for all compounds were determined at the following four concentration levels, expressed as a coefficient of variation (CV): $<6 \%$ for MPA, $<12.3 \%$ for GluMPA, 45 $<11.4 \%$ for AcMPAG and up to $26 \%$ for MPAG. The mean recovery for all of the analytes was as follows: $93.9 \%$ for MPA, $88.3 \%$ for GluMPA, 92.2\% for AcMPAG and 93\% for MPAG.

\section{Statistics}

The analyzed database comprised 249 medical records for 211 patients. The data were weighted according to the number of 53 
1 Table 2. Demographic, clinical, and laboratory characteristics of the study groups: kidney $(n=162)$ and liver transplant recipients $(n=49)$.

\begin{tabular}{|c|c|c|c|c|}
\hline Characteristic & \multicolumn{2}{|c|}{$K t x(n=162)$} & \multicolumn{2}{|r|}{ Ltx $(n=49)$} \\
\hline Age [years] & 47.95 & $(12.1)$ & 50.83 & (12.73) \\
\hline Sex (Female) & 67 & $(41.36 \%)$ & 23 & (46.94\%) \\
\hline BMI $\left[\mathrm{kg} / \mathrm{m}^{2}\right]$ & 25.49 & (3.99) & 25.75 & (3.98) \\
\hline eGFR MDRD $\left[\mathrm{ml} / \mathrm{min} / 1.73 \mathrm{~m}^{2}\right]$ & 44.9 & $(18.18)$ & 59.14 & $(24 / 36)$ \\
\hline $\mathrm{Hb}[\mathrm{g} / \mathrm{dL}]$ & 13.16 & $(1.97)$ & 13.02 & $(2.14)$ \\
\hline WBC [G/L] & 7.81 & $(2.63)$ & 6.45 & $(2.39)$ \\
\hline PLT [G/L] & 223 & $(72-449)$ & 177 & $(9-705)$ \\
\hline AlAT [U/L] & 24 & $(8-294)$ & 41 & $(8-185)$ \\
\hline MPA $[\mu \mathrm{g} / \mathrm{mL}]$ & 2.28 & $(0.31-19.82)$ & 1.06 & $(0.14-5.4)$ \\
\hline GluMPA $[\mu \mathrm{g} / \mathrm{mL}]$ & 0.07 & $(0.004-0.96)$ & 0.03 & $(0.0-0.24)$ \\
\hline AcMPAG $[\mu \mathrm{g} / \mathrm{mL}]$ & 0.77 & $(0.15-7.14)$ & 0.45 & $(0.14-2.84)$ \\
\hline MPAG $[\mu \mathrm{g} / \mathrm{mL}]$ & 70.3 & $(0.66-409.71)$ & 31.44 & $(1.43-168.77)$ \\
\hline MPA corrected dose [mg/day] & 1500 & $(500-2500)$ & 1000 & $(500-2000)$ \\
\hline Time from TX [months] & 58 & $(1-294)$ & 38 & $(1-154)$ \\
\hline $\mathrm{GS}+\mathrm{Tac}+\mathrm{MPA}$ & 60 & (37.04\%) & 21 & $(42.86 \%)$ \\
\hline $\mathrm{GS}+\mathrm{Cs} A+\mathrm{MPA}$ & 55 & $(33.95 \%)$ & 4 & $(8.16 \%)$ \\
\hline $\mathrm{Tac}+\mathrm{MPA}$ & 18 & (11.11\%) & 12 & $(24.49 \%)$ \\
\hline CsA+MPA & 13 & $(8.03 \%)$ & 8 & $(16.33 \%)$ \\
\hline GS+MPA & 11 & $(6.79 \%)$ & 3 & $(6.12 \%)$ \\
\hline$G S+S I R+M P A$ & 3 & $(1.85 \%)$ & & - \\
\hline MPA & 1 & $(0.62 \%)$ & 1 & (2.04\%) \\
\hline $\mathrm{EVE}+\mathrm{MPA}$ & 1 & $(0.62 \%)$ & & - \\
\hline Gastroenterotoxicity & 27 & $(16.67 \%)$ & 8 & $(16.33 \%)$ \\
\hline Infectious complications & 73 & $(45.06 \%)$ & 17 & $(34.7 \%)$ \\
\hline Bacterial infections & 56 & (34.57\%) & 11 & $(22.45 \%)$ \\
\hline Viral infections & 31 & (19.14\%) & 8 & $(16.33 \%)$ \\
\hline
\end{tabular}

BMI - body mass index; TX - type of transplantation; Ktx - kidney transplantation; Ltx - liver transplantation;

eGFR MDRD - estimated glomerular filtration rate calculated by Modification of Diet in Renal Disease equation; AlAT - alanine aminotransferase; MPA - mycophenolic acid; GluMPA - MPA glucoside; AcMPAG - MPA acyl-glucuronide; MPAG - MPA phenolic glucuronide; GS - glucocorticosteroids; CSA - cyclosporine; Tac - tacrolimus; SIR - sirolimus; EVE - everolimus; CMV - cytomegalovirus; WBC - white blood cell count; RBC - red blood cell count; Hb - hemoglobin; PLT - blood platelet count.

records for each patient, such that each unit observation represents one patient. The analyses were corrected by using the Bonferroni correction. Normality was estimated with the 50 Kolmogorov-Smirnov test. Unless specified otherwise, continuous data are described as means \pm SD for a normal distribution, or as medians and ranges for data with any non-normal 53 distribution. The differences between the normally distributed variables were assessed with Student's t test; in other cases, the Mann-Whitney $U$ test was applied. Correlations between the parameters were calculated with the Spearman correlation coefficient for all non-normally distributed values. Then, 50 we used the multivariate analysis methods and a number of regression models were constructed to control for the effects of patients' sex, age, eGFR level and transplant organ on the 53 



$\begin{array}{llllll}80 & 2.00 & 2.20 & 2.40 & 2.60 & 2.80 \\ 1.81 & & & & & 1: \text { MRM of } 2 \text { channels ES+ } \\ \text { TIC (MPA) }\end{array}$
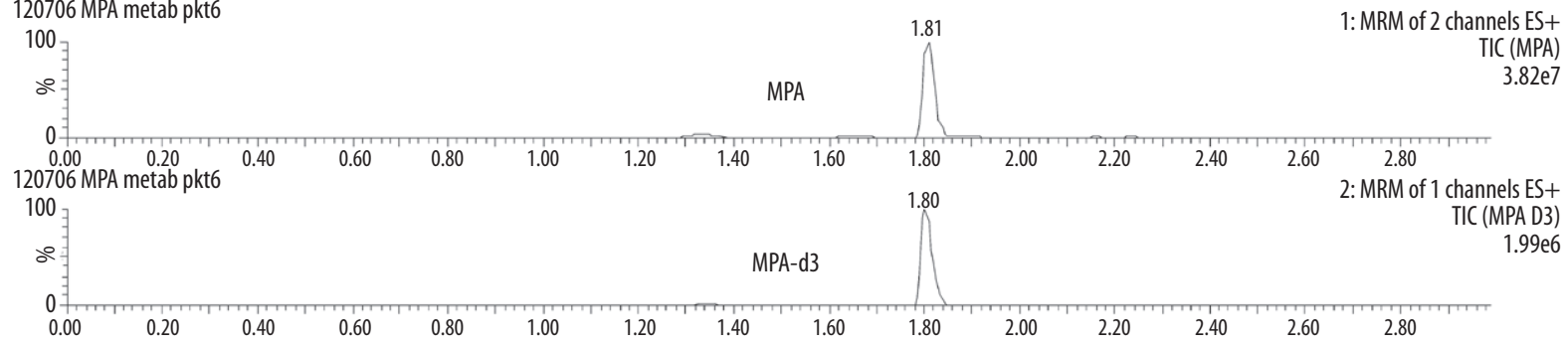

Figure 1. Chromatograms of mycophenolic acid and its derivatives: acyl glucuronide, glucoside, and phenyl glucuronide.

25 concentrations of MPA or its metabolites. Other medical factors, including hemoglobin, red blood cell count (RBC), platelet count (PLT), white blood cell count (WBC), infection and gastroenterotoxicity, were also analyzed. In the latter two cases, in which the dependent variables were binary, logistic regres-

30 sion models were applied; linear regression was used in all other cases. A p-value of $<0.05$ was considered to be significant. All statistical analyses were performed using IBM SPSS Statistics software version 19.0 for Windows.

\section{Results}

Patients' demographics and concentrations of MPA, MPAG, AcMPAG and GluMPA are shown in Table 2. Chromatograms 40 for all compounds in this method are presented in Figure 1.

In the kidney transplant recipients (Ktx) in the group with gastroenterotoxicity (GT) MPA concentrations were higher in comparison with the group without GT (Table 3). There were no 45 significant differences in the MPAG, AcMPAG or GluMPA concentrations between the group with GT and the group with no gastroenterotoxicity in Ktx group (Table 3). Multivariant analysis using logistic regression using gastroenterotoxicity as a dependent variable confirmed that in kidney recipi50 ents MPA concentrations were associated with GT, independent of the patient's sex, age, eGFR, MPA corrected dosage, and metabolite concentrations (Table 4). In liver transplant 53 recipients (Ltx) in the group with GT, there were significantly
MPAG concentrations in comparison with the group with no 25 GT (Table 3). However, there was no difference between those groups in MPA concentrations. Logistic regression in liver patients confirmed negative influence of MPAG concentrations on gastrointestinal adverse effects (Table 4).

Ktx patients with bacterial infections or all types of infections had higher MPAG concentrations in comparison with the group without infections (Table 3). However, logistic regression, including the patients' sex, age, eGFR level, MPA dosage and metabolite concentrations as controlled variables, revealed 35 no significant relationships among MPA, MPAG, AcMPAG and GluMPA and infectious complications (all types of infections, bacterial and viral infections) in this population (Table 4). In liver transplant recipients there was no difference between group with and without infections in MPA or metabolite con- 40 centrations (Table 3). However, multivariant analysis showed the positive influence of AcMPAG on bacterial infections in liver transplant patients (Table 4).

In kidney transplant recipients MPAG and GluMPA was signifi- 45 cantly negatively correlated with hemoglobin (Table 5). Based on the linear regression, MPA or its metabolites did not influence hemoglobin concentration in both Ktx and Ltx patients (Table 4).

Univariate analysis and linear regression using PLT count as a dependent variable revealed no significant relationships among MPA, MPAG, ACMPAG and GluMPAG and PLT counts in 53 
1 Table 3. Comparison of concentrations of MPA and its metabolites in patients with and without adverse effects in the 2 groups: kidney 1 and liver transplant recipients.

5

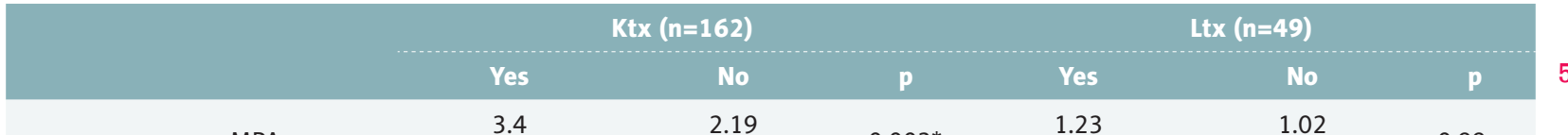

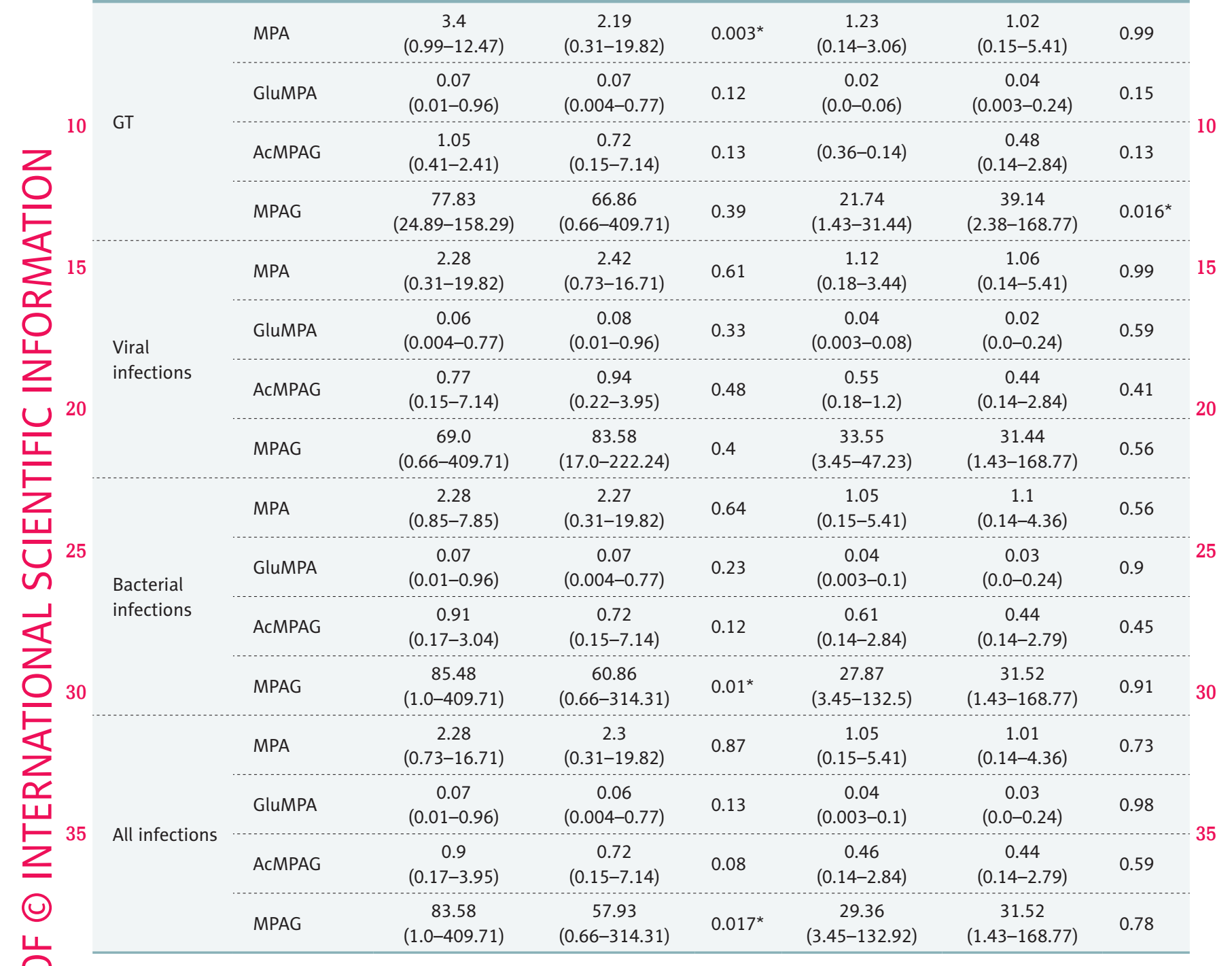

40 GT - gastroenterotoxicity; Ktx: kidney transplantation; Ltx: liver transplantation; MPA: mycophenolic acid; GluMPA: MPA glucoside; 40 AcMPAG: MPA acyl-glucuronide; MPAG: MPA phenolic glucuronide; * $\mathrm{p}<0.05$.

Ktx group (Tables 4, 5). MPAG concentrations were significantly positively correlated with PLT count in Ltx group (Table 5). In 45 the group of liver transplant recipients, we observed a statistically significant positive influence of MPA and a negative influence of GluMPA levels on PLT count after adjusting for patient sex, age, eGFR, AIAT, MPAG, and MPA corrected dosage (Table 4). In kidney and liver transplant recipients there were 50 no associations between MPA and its metabolites and WBC count both in univariate and multivariate analyses (Tables 4, 5).

\section{Discussion}

Here, we have presented an analysis of levels of MPA and its 45 three metabolites in group of MPA-treated transplant recipients. We have used our novel LC-MS/MS method allowing for the complete separation of MPA and its derivatives. We tried to link MPA and its metabolites to side effects in two different groups: kidney and liver graft recipients.

Our analysis revealed higher concentrations of MPA in kidney transplant recipients with gastroenterotoxicity. The relationship 53 
1 Table 4. Relationship between MPA and its metabolites and their therapeutic complications in the 2 groups: kidney and liver transplant recipients (multivariate analyses results).
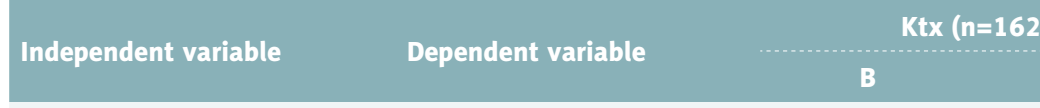

MPA
MPAG
ACMPAG

Gastroenterotoxicity

10

\begin{tabular}{l} 
GluMPA \\
MPA \\
MPAG \\
\hline AcMPAG
\end{tabular}

15

GIUMPA

MPA

MPAG

AcMPAG

Bacterial infections

20

GluMPA

MPA

MPAG

AcMPAG

All types of infections

$$
M P A
$$

30 ACMPAG $\quad$ Hemoglobin

GluMPA

MPA

MPAG

35

AcMPAG

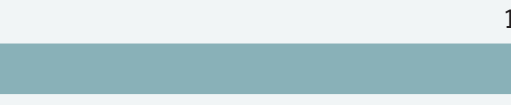

GluMPA

MPA

40

AcMPAG WBC

GluMPA

LTT

.........

0.260

0.04

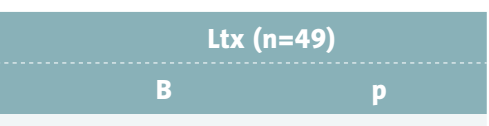

$-0.005$

8.130

0.07

$-0.626$

0.421

$-0.330$

$0.04^{*}$

0.967

0.126

$-0.738$

0.605

0.160

0.625

$-57.113$

0.147

10

0.000

0.060

$-0.012$

0.976

$-0.031$

0.957

$-0.054$

0.096

2.158

0.909

$-0.591$

0.584

$-0.224$

0.269

3.986

0.846

0.093

$-0.531$

0.370

0.004

0.322

0.023

0.158

0.156

0.469

2.040

$0.05^{*}$

2.229

0.254

7.063

0.705

$-0.02$

0.76

$-0.01$

0.97

$0.004 \quad 0.28$

0.003

0.88

$0.19 \quad 0.38$

0.83

0.27

1.6

0.38

$-0.81$

0.96

$\beta$

$\beta$

p

$-0.081$

0.350

$-0.063$

0.804

$-0.134$

0.115

$-0.453$

0.075

0.129

0.108

$-0.193$

0.356

0.071

0.398

$-0.072$

0.801

0.098

0.343

0.650

$0.004^{*}$

0.068

0.499

$-0.001$

0.997

$-0.110$

0.244

0.330

0.087

0.009

0.928

$-0.520$

$0.042^{*}$

$-0.055$

0.522

$-0.083$

0.638

0.047

$-0.078$

0.644

$-0.104$

0.696

0.419

0.128

0.551

Ktx - kidney transplantation; Ltx - liver transplantation; MPA - mycophenolic acid; GluMPA - MPA glucoside; AcMPAG - MPA acylglucuronide; MPAG - MPA phenolic glucuronide; WBC - white blood cell count; PLT - platelet count; * $p<0.05$.

45 between high MPA trough concentrations and adverse drug effects has been reported by several investigators. In a study of 22 kidney transplant recipients, the MPA trough concentration was significantly higher in patients with episodes of diarrhea, infection or hematological adverse effects than in

50 those without such events; these findings are in agreement with the results of the our study [7]. However, other studies have revealed contradictory results in this respect. There was 53 no association between the incidence of GT symptoms and thrombocytopenia and the total and free MPA pharmacokinet- 45 ic parameters in the group of pediatric kidney transplant recipients [8]. In a prospective, randomized, double-blind, multicenter and controlled study of 150 renal transplant recipients, a dose-dependent increase in the adverse effects of kidney recipients was observed in the first 6 months following trans- 50 plantation. However, no relationship between MPA trough concentrations or AUCs and adverse effects was detected [9]. In a retrospective study, 4 of 27 kidney transplant recipients with 53 
1 Table 5. Correlations of MPA and its metabolites concentrations with hemoglobin concentration, blood platelets count, and white blood cells count in the 2 groups: kidney and liver transplant recipients.

5

\begin{tabular}{|c|c|c|c|c|c|}
\hline & & \multicolumn{2}{|c|}{$K t x(n=162)$} & \multicolumn{2}{|c|}{ Ltx $(n=49)$} \\
\hline & & $r$ & p & $r$ & p \\
\hline \multirow{4}{*}{$\mathrm{Hb}$} & MPA & -0.001 & 0.99 & 0.21 & 0.16 \\
\hline & GluMPA & -0.19 & $0.015^{*}$ & 0.09 & 0.54 \\
\hline & AcMPAG & -0.02 & 0.8 & 0.03 & 0.86 \\
\hline & MPAG & -0.28 & $0.0003^{*}$ & -0.09 & 0.55 \\
\hline \multirow{4}{*}{ Plt } & MPA & 0.05 & 0.54 & 0.24 & 0.09 \\
\hline & GluMPA & 0.06 & 0.43 & 0.25 & 0.09 \\
\hline & AcMPAG & -0.02 & 0.77 & 0.15 & 0.3 \\
\hline & MPAG & 0.07 & 0.4 & 0.32 & $0.02^{*}$ \\
\hline \multirow{4}{*}{ WBC } & MPA & -0.01 & 0.92 & 0.09 & 0.54 \\
\hline & GluMPA & 0.05 & 0.57 & 0.06 & 0.67 \\
\hline & AcMPAG & 0.02 & 0.8 & 0.05 & 0.72 \\
\hline & MPAG & 0.11 & 0.15 & 0.004 & 0.98 \\
\hline
\end{tabular}

Ktx - kidney transplantation; Ltx - liver transplantation; MPA - mycophenolic acid; GluMPA - MPA glucoside; AcMPAG - MPA acylglucuronide; MPAG - MPA phenolic glucuronide; Plt - blood platalets; $\mathrm{Hb}$ - hemoglobin; WBC - white blood cells; * $p<0.05$.

gastrointestinal adverse effects had significantly lower MPA 25 AUC values. It was suggested that, in patients with gastrointestinal toxicity, drug absorption is decreased, leading to further local irritation [10].

Some authors have postulated the involvement of AcMPAG in 30 causing gastrointestinal disturbances among patients treated with mycophenolate mofetil or sodium [11]. We did not observe such associations in kidney recipients. Other studies were also unable to confirm the relationship between MPA metabolites and GT: Grinyo et al. did not find correlations between 35 MPA, AcMPAG exposure or maximal concentration (C max) of ACMPAG and the occurrence of gastrointestinal symptoms after 7 days and after 1 month in their pharmacokinetic study of 82 renal transplant recipients [12]. Heller et al. were not able to find any relationship between AcMPAG concentrations and 40 the incidence of diarrhea in renal transplant recipients [13].

Little is known about MPA metabolites and their associations with gastrointestinal problems in liver transplant recipients. We have found a negative influence of MPAG levels on gastro45 enterotoxicity in liver transplant recipients. The clinical significance of this finding remains unclear. It cannot be excluded that lower MPAG levels are the consequence of decreased enterohepatic circulation in the course of diarrhea. Interestingly, MPA concentrations were not related to gastrointestinal symp-

50 toms in this group. In a study of 67 liver transplant patients the occurrence of diarrhea was not related to pharmacokinetics of MPA and its metabolites [14].
There are few reports available on the impact of the concentrations of MPA and its metabolites on the incidence of infec- 25 tious complications in SOT patients. It has been proposed that AcMPAG may contribute to side effects of the MPA formulations, which include myelotoxicity, infections and gastroenterotoxicity [15]. Those toxic effects may be mediated by interleukin- 6 and tumor necrosis factor alpha induced by AcMPAG in human 30 mononuclear leukocytes [11]. Interestingly, it has been demonstrated that some of the effects of AcMPAG are independent of the major mechanism of MPA action, which is associated with inosine monophosphate dehydrogenase inhibition [16]. It has been speculated that AcMPAG could exert an antipro- 35 liferative effect and inhibit proliferation of human mononuclear leukocytes via a mechanism independent of guanosine triphosphate (GTP) depletion [15]. Other studies have shown contradictory results regarding the influence of MPA levels on infectious complications. The mean MPA through concentra- 40 tions were higher in 13 patients with adverse effects, mainly infectious, compared with those without adverse effects in a retrospective study of 30 renal transplant recipients [17]. On the other hand, in a retrospective study of 21 renal transplant patients over the first 28 days following transplantation, 45 no differences in the drug dosage or MPA AUC were detected between recipients with and without viral infections [18]. Some authors reported relationship between high free MPAAUC and MPA-Cmax levels and infections [8]. The authors did not observe such a relationship when the total MPA pharma- 50 cokinetic parameters were analyzed. 
1 In our study in kidney transplant recipients neither MPA nor its metabolites influenced bacterial or viral infections' occurrence. However, in liver transplant recipients unfavorable influence of AcMPAG on bacterial infections' incidence was ob-

5 served. We have not found any reports concerning relationship among MPA, its metabolites and infection episodes in Ltx patients. This finding seems to be interesting and suggests the need of further research on this topic.

10 In multivariate analyses we did not confirmed negative correlations of GluMPA and MPAG concentrations and hemoglobin levels in Ktx patients. However, other authors observed a negative influence of MPA, MPAG and AcMPAG on erythropoiesis. In a prospective study of 100 renal transplant patients,

15 those with anemia and leucopenia had significantly higher MPA AUC12 than those with normal RBC and WBC [19]. A prospective multicenter study of 33 kidney transplant patients demonstrated a relationship of high MPAG and AcMPAG levels with leucopenia and anemia [20]. In the group of 106 renal trans-

20 plant recipients, the MPAG pharmacokinetic parameters correlated negatively with hemoglobin and hematocrit [21]. The authors of this paper concluded that MPAG might be a predicting factor for the side effects of mycophenolates. Ting et al. reported that anemia, leucopenia, infection and rejection 25 occurred in lung and heart transplant recipients with higher ACMPAG AUC [22]. Inconclusive results of the research to date suggest that also in this field there is a need of analyses of larger patients' groups. In liver transplant recipients we did not observe the influence of MPA metabolites on hemoglobin

30 levels. We did not also find any reports on this issue in the literature. We hope to obtain more conclusive results from analyses of larger group, which is currently recruited by our team.

In kidney transplant recipients we found no associations be35 tween MPA or its metabolites and thrombocytopenia. It is consistent with observations made by other investigators. Weber et al. did not observe any associations between free or total MPA pharmacokinetic parameters and thrombocytopenia in kidney transplant recipients [8]. In liver transplant patients

40 GluMPA was associated with thrombocytopenia, suggesting a disadvantageous effect of GluMPA on thrombocytopoiesis in the bone marrow. Additionally, higher MPA levels were associated with higher Plt count, which was confirmed by multivariate analyses. The significance of this finding remains elu45 sive and needs further investigation.

The relationship between MPA levels and gastrointestinal symptoms, observed in kidney transplant patients, was not confirmed in the liver transplant group, possibly because the

50 number of patients in this group was too small. The fact that the association between MPA metabolites and other adverse effects occurred only in liver transplant recipients is interest53 ing. We hypothesized that it might be caused by lower eGFR values in the Ktx group in comparison with liver transplant re- 1 cipients. It could result in increased accumulation of MPA metabolites and cause attenuation of interplay between them and the analyzed complications.

The present study has some limitations, including its observational nature and limitation to a single center. The lack of exclusion criteria reflects the natural characteristics of this patient population after solid organ transplantation. The heterogeneity of the group is also left open to being influenced by 10 confounding factors, such as various lengths of time elapsed since transplantation, 2 different MPA prodrugs, and different immunosuppression regimens. It has been proved that there are some differences between MMF and MPS preparations in terms of maximal concentration and AUC profiles, but their 15 impact on IMPDH activity is not well defined [23,24]. In pediatric liver transplant patients there was no statistically significant difference between formulations of MPA in the gene expression of IMPDH 2, in the AUC(0-12h), or in C max, but peak concentration occurred later with MPS [25]. Parallel analysis 20 of the concentrations of MPA and its metabolites in patients after Ktx and Ltx allowed a unique comparison of differences in their impact on adverse reactions occurrence between kidney and liver transplant recipients.

\section{Conclusions}

In conclusion, there are differences in the relationship between concentrations of MPA or its metabolites and adverse effects 30 in kidney graft recipients and liver graft recipients. Those differences only partially could be explained. Precise measurements of the total MPA trough concentrations seem to be important in kidney recipients to monitor adverse gastrointestinal effects. On the other hand, the quantification of AcMPAG con- 35 centrations in liver transplant recipients receiving immunosuppressive therapy with MPA may be helpful in avoiding bacterial infections. Additionally, GluMPA, of unknown significance so far, seems to have a toxic effect on thrombopoiesis in this group. The assessment of the GluMPA concentrations could 40 help to prevent thrombocytopenia. Further studies are needed to verify if monitoring of MPA and its metabolites would be beneficial for the long-term management of patients receiving mycophenolate formulations. The quantification of MPA metabolites could then be an important part of therapeutic 45 monitoring and may be helpful in establishing safer immunosuppressive therapies.

\section{Acknowledgments}

The authors thank Roche Poland for kindly donating standards of mycophenolic acid metabolites. 


\section{References:}

1. Franklin TJ, Cook JM: The inhibition of nucleic acid synthesis by mycophenolic acid. Biochem J, 1969; 113: 515-24

2. Vanhove T, Kuypers D, Claes KJ et al: Reasons for dose reduction of mycophenolate mofetil during the first year after renal transplantation and its impact on graft outcome. Transpl Int, 2013; 26(8): 813-21

3. Shipkova M, Armstrong VW, Weber L et al. Pharmacokinetics and protein adduct formation of the pharmacologically active acyl glucuronide metabolite of mycophenolic acid in pediatric renal transplant recipients. Ther Drug Monit, 2002; 24(3): 390-99

4. Shipkova M, Armstrong VW, Wieland E et al: Identification of glucoside and carbonyl-linked glucuronide conjugates of mycophenolic acid in plasma of transplant recipients treated with mycophenolate mofetil. Br J Pharmacol, 1999; 126(5): 1075-82

5. Schuetz E, Shipkova M, Armstrong VW et al: Identification of a pharmacologically active metabolite of mycophenolic acid in plasma of transplant recipients treated with mycophenolate mofetil. Clin Chem, 1999; 45(3): 419-22

6. Levey AS, Bosch JP, Lewis JB et al: A more accurate method to estimate glomerular filtration rate from serum creatinine: a new prediction equation. Modification of Diet in Renal Disease Study Group. Ann Intern Med, 1999; 16: $461-70$

7. Lu YP, Lin B, Liang MZ et al: Correlation of mycophenolic acid pharmacokinetic parameters with side effects in Chinese kidney transplant recipients treated with mycophenolate mofetil. Transpl Proc, 2004; 35(7): 2079-81

8. Weber LT, Shipkova M, Armstrong VW et al: The pharmacokinetic-pharmacodynamic relationship for total and free mycophenolic acid in pediatric renal transplant recipinets: a report of theGerman study group on mycophenolic mofetil therapy. Am J Soc Nephrol, 2002; 13: 759-68

9. van Gelder T, Hilbrands LB, Vanrenterghem $Y$ et al: A randomized doubleblind, multicenter plasma concentration controlled study of the safety and efficacy of oral mycophenolate mofetil for the prevention of acute rejection after kidney transplantation. Transplantation, 1999; 68: 261-66

10. Pillans PI, Rigby RJ, Kuble P et al: A retrospective analysis of mycophenolic aid and cyclosporine concentrations with acute rejection in renal transplant recipients. Clin Biochem, 2001; 34(1): 77-81

11. Wieland E, Shipkova M, Schellhaas $U$ et al: Induction of cytokine release by the acyl glucuronide of mycophenolic acid: a link to side effects? Clin Biochem, 2000; 33: 107-13

12. Grinyo JM, Ekberg H, Mamelok RD et al: The pharmacokinetics of mycophenolate mofetil in renal transplant recipients receiving standard-dose or low-dose cyclosporine, low-dose tacrolimus or low-dose sirolimus: the Symphony pharmacokinetic substudy. Nephrol Dial Transplant, 2009; 24 2269-76

13. Heller T, van Gelder T, Budde K et al: Plasma concentrations of mycophenolic acid acyl glucuronide are not associated with diarrhea in renal trabsplant recipients. Am J Transplant, 2007; 7: 1822-31

14. Xia ZW, Jun CY, Hao C et al: The occurence of diarrhea is not related to the pharmacokinetics of MPA and its metabolites in liver transplant patients. Eur J Clin Pharmacol, 2010; 66(7): 671-79

15. Shipkova M, Wieland E, Schutz E et al: The acyl glucuronide metabolite of 5 mycophenolic acid inhibits the proliferation of human mononuclear leukocytes. Transpl Proc, 2001; 33: 1080-81

16. Petrova DT, Heller T, Hitt R et al: Regulation of IL2 and NUCB1 in mononuclear cells treated with acyl glucuronide of mycophenolic acid reveals effects independent of inosine monophosphate dehydrogenase inhibition. Ther Drug Monit, 2009; 31: 31-41

17. Hubner GI, Eismann R, Sziegoleit W et al: Relationship between mycophe- 10 nolate mofetil side effects and mycophenolic acid plasma trough levels in renal transplant patients. Arzneimitt Forsch, 2000; 50(10): 936-40

18. Satoh S, Tada H, Murakami $M$ et al: The influence of mycophenolate mofetil versus azathioprine and mycophenolic acid pharmacokinetics in the incidence of acute rejection and infectiouc complications after renal transplantation. Transpl Proc, 2005; 37(4): 1751-53

19. Kuypers DR, Claes K, Evenepoel P et al: Clinical efficacy and toxicity pro- 15 file of tacrolimus and mycophenolic acid in relation to combined long-term pharmacokinetics in de novo renal allograft recipients. Clin Pharmacol Ther, 2004; 75(5): 343-47

20. Kuypers DR, Vanrenterghem Y, Squifflet JP et al: Twelve-month evaluation of the clinical pharmacokinetics of total and free mycophenolic acid and its glucuronide metabolites in renal allograft recipients on low dose tacrolimus in combination with mycophenolate mofetil. Ther Drug Monit, 2003; 20 25(5): 609-22

21. Sobiak J, Kaminska J, Glyda $M$ et al: Effect of mycophenolate mofetil on hematological side effects indicence in renal transplant recipients. Clin Transplant, 2013; 27(4): E407-14

22. Ting LS, Benoit-Biancamano MO, Bernard O et al: Pharmacogenetic impact UDP-glucuronosyltransferase metabolic pathway and multidrug resistance- 25 associated protein 2 transport pathway on mycophenolis acid in thoracic transplant recipients: an exploratory study. Pharmacotherapy, 2010; 30(11): 1097-108

23. Tornatore KM, Sudchada P, Attwood K et al: Race and drug formulation influence on mycophenolic acid pharmacokinetics in stable renal transplant recipients. J Cli Pharm, 2013; 53(3): 285-93

24. Budde K, Tedesco-Silva H, Pestana JM et al: Enteric-coated mycophenolate 30 sodium provides higher mycophenolic acid predose levels compared with mycophenolate mofetil: implications for therapeutic drug monitoring. Ther Drug Monit, 2007; 29(3): 381-84

25. Reyes $\mathrm{H}$, Hernandez AM, Valverde $\mathrm{S}$ et al: Efficacy and safety of conversion of mycophenolate mofetil to enteric-coated mycophenolate sodium in Mexican renal transplant children. Pediatr Transplant, 2010; 14(6): 746-52 